Development of Durable 3-Electrode Lithium-Ion Pouch Cells with LTO Reference Mesh: Aging and Performance Studies

To cite this article: Bernd Epding et al 2019 J. Electrochem. Soc. 166 A1550

View the article online for updates and enhancements. 


\title{
Development of Durable 3-Electrode Lithium-Ion Pouch Cells with LTO Reference Mesh: Aging and Performance Studies
}

\author{
Bernd Epding, ${ }^{1, z}$ Agate Broda, ${ }^{2}$ Björn Rumberg, ${ }^{1}$ Hannes Jahnke, ${ }^{1}$ and Arno Kwade ${ }^{3}$ \\ ${ }^{I}$ Volkswagen AG, Group Research, 38436 Wolfsburg, Germany \\ ${ }^{2}$ Leibniz Universität Hannover, Electric Energy Storage Systems, 30167 Hannover, Germany \\ ${ }^{3}$ Technische Universität Braunschweig, Institute for Particle Technology, 38104 Braunschweig, Germany
}

\begin{abstract}
Building a lithium-ion cell with a third reference electrode which is stable and does not disturb the cell characteristics promises deep insights into the cell performance and aging behavior. In this study, a lithium titanium oxide coated aluminum mesh is introduced into lab size $28 \mathrm{mAh}$ pouch cells, which allows the cells to be braced as usual. The influence of inserting the coated mesh together with an additional separator is analyzed using electrochemical impedance spectroscopy and cyclic aging tests. Cells with this reference electrode show small deviations in the Nyquist plot and an increased capacity fade compared to the standard cells. Nonetheless, these 3-electrode cells already allowed C-rate performance tests in a fresh and aged cell state. According to this, the charge current profiles for fresh and aged cells are optimized as step wise protocols to improve the utilization of the anode overpotential reserve while avoiding lithium plating. In order to realize this, the aged cells require a $60 \%$ slower protocol. This work shows that the overpotentials on the anode side increase during aging, which makes the cells more susceptible to lithium plating. However, compared to a constant current charge, a reduction in charging time of $30 \%$ is possible.

(C) 2019 The Electrochemical Society. [DOI: 10.1149/2.0851908jes]
\end{abstract}

Manuscript submitted February 25, 2019; revised manuscript received April 2, 2019. Published May 7, 2019.

Over the last years it has been a prime goal to increase the energy density of lithium-ion cells to facilitate longer range of battery electric vehicles or longer usage times of mobile devices while maintaining the package volume. By now it has become clear that with state of the art cells $100 \mathrm{kWh}$ batteries for electric vehicles can be achieved and driving ranges of over $500 \mathrm{~km}$ are possible. Still, to achieve maximum flexibility for the user, the battery needs to be able to be recharged quickly. With state of the art lithium-ion technology, the charge capability of a cell tends to decrease with increasing energy density. ${ }^{1}$ Since the current density in the cell increases for higher energy density cells, the diffusion in the thick porous electrodes limits lithium-ion transport. This in turn leads to increasing overpotentials at the anode surface which eventually result in a negative electrode potential vs. $\mathrm{Li} / \mathrm{Li}^{+} .{ }^{2}$ Whenever this is the case, the deposition of lithium as a metal film on the graphite surface becomes thermodynamically possible, so called lithium plating. ${ }^{3-5}$ Lithium plating can lead to a fast capacity loss since the metallic lithium reacts with the electrolyte to form new passivating layers or is no longer available for the electrochemical reactions due to loss of electrical contact to the graphite. ${ }^{6}$ If the lithium plating appears in the form of dendrites which pierce through the separator, it can lead to electrical shorts inside the battery causing an accelerated self-discharge or even thermal runaway. ${ }^{3}$ To be able to use current cell technologies to their maximum capabilities while keeping the operation safe and to compare different approaches to overcome their limitations, new methods are needed to precisely detect the occurrence of lithium plating. Various methods have been described in the literature to directly or indirectly detect lithium plating in lithium-ion cells. ${ }^{3}$ So far none of these methods allow the direct detection of lithium plating without modifying the cell setup or disassembling it to gather electrode material. Indirect electrochemical techniques (lithium stripping, voltage relaxation, high precision coulometry $)^{6-8}$ can indicate that lithium plating occurred during the previous charge step. However, in order to detect the precise onset of lithium plating during a charge period, many cells have to be tested under varying external conditions. Furthermore, testing multiple conditions with the same cell reduces data quality since massive lithium deposition is required before it can be detected with indirect methods, which severely damages the cell. Also, the fast reintercalation of lithium into the graphite anode limits the plating detectability at higher temperatures which are commonly induced by fast charging. ${ }^{9-11}$ Similar arguments can be made toward methods like neutron diffraction ${ }^{12}$ or volume detection ${ }^{13}$ but here, actual production cells can be tested. More sensitive techniques like EPR ${ }^{14}$ or $\mathrm{NMR}^{15}$ still require small specialized cells to fit into the measurement equipment.

${ }^{\text {z} E-m a i l: ~ b e r n d . e p d i n g @ ~ v o l k s w a g e n . d e ~}$
Finally, inserting a third electrode as a reference into lithium-ion cells has already proven to give great insight into their properties. With a reference electrode, anode and cathode potential can be measured independently and thus questions like proper balancing of electrodes and impedance contributions can be checked. Due to the fact that the anode potential falls below $0 \mathrm{~V} \mathrm{vs} \mathrm{Li} / \mathrm{Li}^{+}$before lithium plating occurs, a reference electrode can also be used to detect its onset during a charge period. ${ }^{3-5}$ Additionally, it gives the opportunity to stop a charge step if the anode potential reaches a critical value and thus limits the damage to the test cell. This way a single cell can be used to test a whole range of operating conditions.

The optimal way to construct a 3-electrode lithium-ion cell is still under debate. Depending on the intended measurements many possible designs exist. For electrochemical impedance spectroscopy (EIS) measurements the geometry of the cell design can introduce measurement artifacts. ${ }^{16}$ Furthermore, the transferability of results gathered from 3 -electrode cells to the actual production cells is often questionable. If the cell design has to be changed a lot to insert the reference electrode or if the reference has an inconvenient geometry and/or is placed at the wrong position inside the cell, the obtained electrochemical results do not allow precise conclusions for production cells. ${ }^{17}$ Finally, most 3-electrode designs based on lithium metal electrodes suffer from fast degradation since lithium metal reference electrodes change or are even consumed in the cell. ${ }^{18}$ Thus, no continuous aging information is available from 3-electrode cells but only spot tests at certain states of aging are possible. Furthermore, it is difficult to build 3 -electrode cells from post-mortem material of aged cells as the electrodes often show inhomogeneous aging patterns and become brittle. ${ }^{4}$

In this work a 3-electrode pouch cell design is realized in which the reference is an aluminum mesh coated with lithium titanium oxide (LTO, $\mathrm{Li}_{4} \mathrm{Ti}_{5} \mathrm{O}_{12}$ ) similar to what was shown by Costard et al. for small coin cell types. ${ }^{19}$ The mesh has the same size and geometry as the electrodes and allows the 3-electrode pouch cells to be braced the same way as standard pouch cells. LTO serves as a chemically stable two phase reference material. ${ }^{20,21}$ This way it is possible to show the influence of the changes made to the standard cell on the aging behavior and gather continuous 3 -electrode data over the whole aging campaign. C-rate tests were conducted on fresh as well as aged 3 -electrode cells. The results indicate that the onset of lithium plating is heavily influenced by the aging of the electrode materials as it was predicted by Yang et al. and observed by others. ${ }^{22-24}$ Thus, common fast charge protocols which are defined for fresh cells will likely lead to lithium plating in aged cells. Finally, a current step protocol is derived for the 3-electrode cells to determine how far the charging time can be reduced with an optimized charging protocol in fresh and aged cells. 


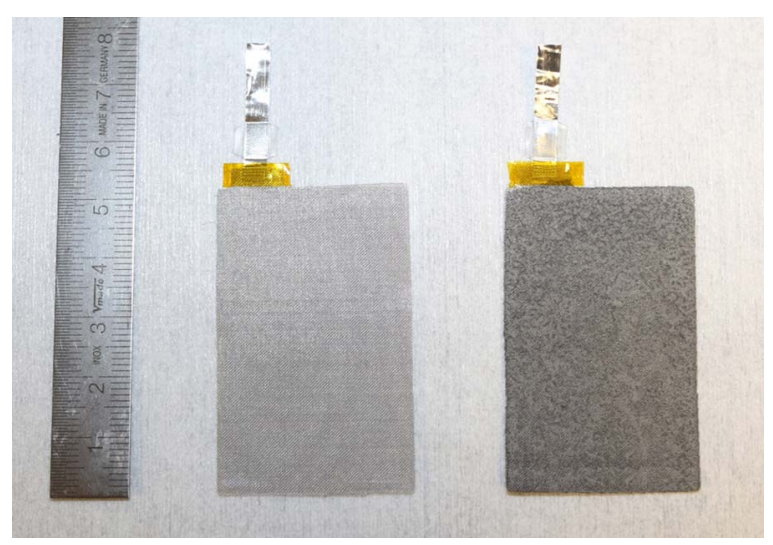

Figure 1. LTO reference electrode. Uncoated aluminum mesh (left) with current collector tap already welded on. Aluminum mesh after dip coating with LTO (right).

\section{Experimental}

Production of LTO slurry and coating on aluminum mesh.For the reference electrodes LTO was coated onto a woven aluminum mesh (SPÖRL KG) with $52 \mu \mathrm{m}$ thick wires and $75 \mu \mathrm{m}$ mesh opening. The LTO slurry consisted of $25.5 \mathrm{~g}$ LTO powder with about $7 \mu \mathrm{m} \mathrm{LTO}$ particles, $1.5 \mathrm{~g} \mathrm{C} 65$ conductive carbon black and $3 \mathrm{~g}$ Seika AG-binder dispersed in $200 \mathrm{ml}$ deionized water at $2000 \mathrm{rpm}$ for 15 minutes. Aluminum mesh sheets $(3.25 \mathrm{~cm} \times 5.15 \mathrm{~cm})$ were punched out and an aluminum current collector was welded on. The mesh was cleaned thoroughly with isopropyl alcohol and manually dip coated in the LTO slurry. Following this, the meshes were dried in air for 1 hour and excess slurry was carefully removed at the bottom of the meshes. Finally, the meshes were dried at $100^{\circ} \mathrm{C}$ for 2.5 hours in vacuum.

Building and characterizing coin cells with LTO mesh against lithium metal.-In order to check the equilibrium voltage as well as the capacity of the produced LTO meshes, $14 \mathrm{~mm}$ diameter samples were prepared as described above and transferred into an argon filled glove box. With these electrodes CR2032 coin cells were built using a lithium metal counter electrode, two layers of a Celgard 2500 separator and $70 \mu \mathrm{l}$ standard electrolyte containing carbonate solvents and $\mathrm{LiPF}_{6}$.

The coin cells were discharged with a current of $50 \mu \mathrm{A}$ until a cutoff voltage of $1.3 \mathrm{~V}$ was reached using a BaSyTec CTS test bench. Afterwards, the cell voltage was monitored over a 12 day period. Testing was performed at room temperature $\left(23^{\circ} \mathrm{C} \pm 1^{\circ} \mathrm{C}\right)$.

Building and characterizing pouch cells with and without LTO reference mesh.-Lab-size nominal $28 \mathrm{mAh}$ pouch cells were fabricated using one-side machine coated electrodes for PHEV applications from the Volkswagen-Varta Microbattery joint venture. The electrodes employ NMC622 on the cathode side and graphite on the anode side. Sheets of $3.1 \mathrm{~cm} \times 5 \mathrm{~cm}$ for the cathodes and $3.25 \mathrm{~cm} \times 5.15 \mathrm{~cm}$ for the anodes were punched out of the electrode coils. Small areas at the top of each electrode were cleaned manually from active material and current collector tabs were welded on via ultrasound. The electrodes were dried overnight in vacuum at room temperature and subsequently at $100^{\circ} \mathrm{C}$ (anodes) or $150^{\circ} \mathrm{C}$ (cathodes) in vacuum for 5 hours before cell assembly.

Care was taken to prevent a short between the cathode and the reference electrode by isolating the current collector tabs with Kapton tape on the inside of the cell (see Figure 1) and with scotch tape on the outside since both taps exit the cell at the same position to make sealing easier. After the assembly of the various cell configurations with separators (W-Scope) shown in Figure 2, the cells were dried again at $60^{\circ} \mathrm{C}$ in vacuum overnight. Following this, the cells were transferred into an argon filled glove box, injected with 280-350 $\mu$ l (depending on the number of layers) of a standard electrolyte containing carbonate solvents and $\mathrm{LiPF}_{6}$ and finally sealed. After tempering at $60^{\circ} \mathrm{C}$ for (a)

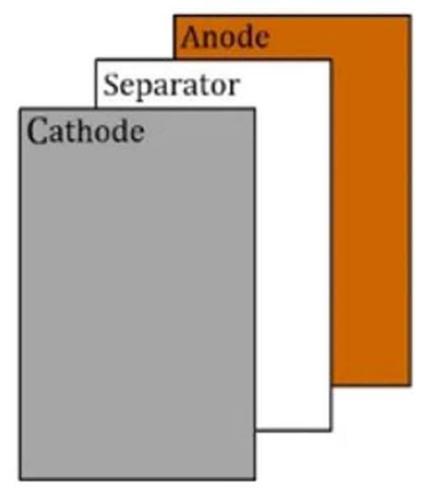

(b)

(c)
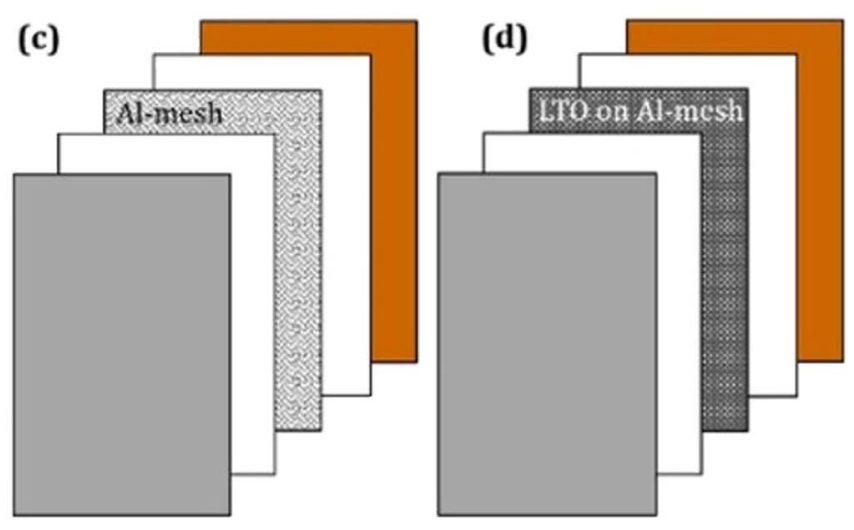

Figure 2. Incremental changes to the pouch cell design. Cells were built with: a) one layer of separator (standard cell), b) two layers of separator, c) an uncoated aluminum mesh between two layers of separator, d) an aluminum mesh coated with LTO between two separators between the anode and the cathode.

2 hours the cells were degassed and sealed again. The finished pouch cells were braced in plastic fixtures $(0.4 \mathrm{Nm}$ torque $)$ and connected to a BaSyTec CTS test bench for formation.

All electrical tests involving the 3-electrode cells and any electrochemical impedance spectroscopies were performed using a Biologic VMP3 potentiostat. Cycling of cells without a reference electrode was done using the BaSyTec CTS test bench. The cells were analyzed either at room temperature $\left(23^{\circ} \mathrm{C} \pm 1^{\circ} \mathrm{C}\right)$ or in a CTS climate chamber $\left(45^{\circ} \mathrm{C} \pm 1{ }^{\circ} \mathrm{C}\right)$.

Formation consisted of a C/20 charge step to $4.15 \mathrm{~V}$ followed by a $\mathrm{C} / 20$ discharge for 5 hours, $\mathrm{C} / 20$ charge to $4.15 \mathrm{~V}$ and again 5 hour $\mathrm{C} / 20$ discharge. This was followed by a $\mathrm{C} / 10$ charge to $4.15 \mathrm{~V}$, a C/10 discharge for 5 hours, $\mathrm{C} / 10$ charge to $4.15 \mathrm{~V}$ and then $\mathrm{C} / 10$ discharge to $3.0 \mathrm{~V}$ to measure the initial capacity. Following this, a $\mathrm{C} / 3$ charge to $4.15 \mathrm{~V}$ with a constant voltage $(\mathrm{CV})$ phase to $\mathrm{C} / 20$ and a $\mathrm{C} / 3$ discharge without $\mathrm{CV}$ phase to $3.0 \mathrm{~V}$ were performed. The formation ended with a C/3 charge to $3.6 \mathrm{~V}$ (about $20 \%$ SoC) to avoid an unintended deep-discharge. Between every charge and discharge step a $10 \mathrm{~min}$ rest period was applied. Using formation cycles at high cell voltages instead of full cycles allowed us to shorten the procedure and to form a stable, compact solid electrolyte interface (SEI) from the beginning. ${ }^{25}$

After the cell formation, the reference was conditioned by connecting the reference electrode to the cathode. The reference potential was set by charging $1 \mathrm{mAh}$, which is about one sixth of its theoretical capacity, into the LTO electrode with a current of $0.5 \mathrm{~mA}$ to reach the stable two phase voltage plateau.

Aging cycles were conducted at $45^{\circ} \mathrm{C}$. The cells were charged with $3 \mathrm{C}$ to $4.15 \mathrm{~V}$ followed by a $\mathrm{CV}$ phase to $\mathrm{C} / 20$. After $10 \mathrm{~min}$ rest time the cells were discharged with $3 \mathrm{C}$ to $3.0 \mathrm{~V}$ without $\mathrm{CV}$ phase followed again by $10 \mathrm{~min}$ rest time. 
Table I. Initial capacity and characteristic resistance of all built cell types as mean values with corresponding corrected standard deviation.

$$
\text { Cell type }
$$

Initial $\mathrm{C} / 10$ capacity [mAh]
Initial characteristic resistance $[\Omega]$

$\begin{array}{ll}31.74 \pm 1.25 & 1.20 \pm 0.04 \\ 32.69 \pm 0.26 & 1.45 \pm 0.08 \\ 32.54 \pm 0.32 & 1.50 \pm 0.06 \\ 32.07 \pm 0.71 & 1.69 \pm 0.04\end{array}$

In order to evaluate the state of health of all the cells, a checkup routine at $23^{\circ} \mathrm{C}$ was performed after formation, before and after conducting the electrochemical tests and after 250 respectively 500 aging cycles. First, at a cell SoC of $20 \%$, the LTO electrodes were conditioned again by discharging the LTO against NMC to a voltage of $0.8 \mathrm{~V}$ and recharging $1 \mathrm{mAh}$ into the LTO electrode. Then, three full $1 \mathrm{C}$ cycles were performed with a $\mathrm{CV}$ phase only in charge direction until a current of $\mathrm{C} / 20$. Between every charge end discharge step a 30 minute rest period was applied. After the last $1 \mathrm{C}$ constant current (CC) discharge the cells were charged with $\mathrm{C} / 3$ to $3.6 \mathrm{~V}$ (about $20 \%$ SoC). Following a rest period overnight, electrochemical impedance spectra were measured in a frequency range between $10 \mathrm{mHz}$ and $100 \mathrm{kHz}$.

The fast charge capabilities of the cells were measured using asymmetric C-rate tests at $23^{\circ} \mathrm{C}$. In this case the charge current was varied $(\mathrm{C} / 5, \mathrm{C} / 2,1 \mathrm{C}, 2 \mathrm{C}, 3 \mathrm{C}, 4 \mathrm{C})$ while the discharge current was always $1 \mathrm{C}$. Three identical cycles were performed before the next charge current was applied. The charge step was terminated when the maximum voltage of $4.15 \mathrm{~V}$ was reached. No CV phases were used so that the cells were not damaged unnecessarily. Between every charge and discharge step a 30 minute rest period was applied. For the 3 -electrode cells, the charge step was additionally terminated if the anode potential reached $-1.562 \mathrm{~V}$ vs. LTO (respectively $0 \mathrm{~V}$ vs. $\mathrm{Li} / \mathrm{Li}^{+}$) to avoid damage from lithium plating.

\section{Results and Discussion}

Quality of the LTO reference mesh.-The suitability of the produced LTO electrodes as reference electrodes was studied by the coin cell measurements against lithium metal and by scanning electron microscope (SEM) imaging (see Figures $3 \mathrm{a}$ and $3 \mathrm{~b}$ ). Coin cell experiments demonstrated a good agreement of the LTO electrode capacity with theoretical values derived from weight measurements. Furthermore, the stable potential of $1.562 \mathrm{~V}$ vs $\mathrm{Li} / \mathrm{Li}^{+}$, as shown in Figure $3 \mathrm{c}$, is in good agreement with LTO potential values published by others. ${ }^{19,21}$ From the SEM images it becomes clear that the coarse LTO particles developed for standard electrode manufacturing are not optimal for coating a reference mesh. Most of the mesh openings are filled with LTO particles while the outside surfaces of the aluminum wires are hardly coated. However, at higher magnification the porous structure of the LTO becomes visible which allows lithium to readily diffuse through the mesh structure. Since most of the LTO is inside the mesh openings, the thickness of the mesh does not increase much further and an even electrode is obtained which does not damage the separator. Still, the use of LTO nanoparticles, as demonstrated by Costard et al., should be preferred for future experiments. ${ }^{19}$

Reproducibility of pouch cell manufacturing.-Several cells were built for each cell type (see Figure 2). To demonstrate reproducibility, Table I shows the C/10 (2.8 mA) cell capacity after formation for each type as well as their initial characteristic resistance (the local minimum in the Nyquist plot) derived from EIS measurements shown in Figure 4a.

Incremental changes to cell design.-The influence of the changes to the standard cell design as introduced by the insertion of the LTO reference mesh between the anode and cathode was evaluated by EIS measurements. In Figure 4 the EIS results for standard cells, cells with two separator sheets, cells with two separators and an uncoated aluminum mesh and complete 3-electrode cells with LTO coated aluminum meshes are compared. Figure 4 a shows the impedance spectra in a Nyquist plot. An increasing shift of the spectra to higher real impedance values is observed when inserting additional layers between the anode and the cathode. This corresponds to an increasing internal resistance due to longer diffusion paths in the electrolyte. ${ }^{26,27}$ The small spread of the spectra for nominally identical cells is caused

a)

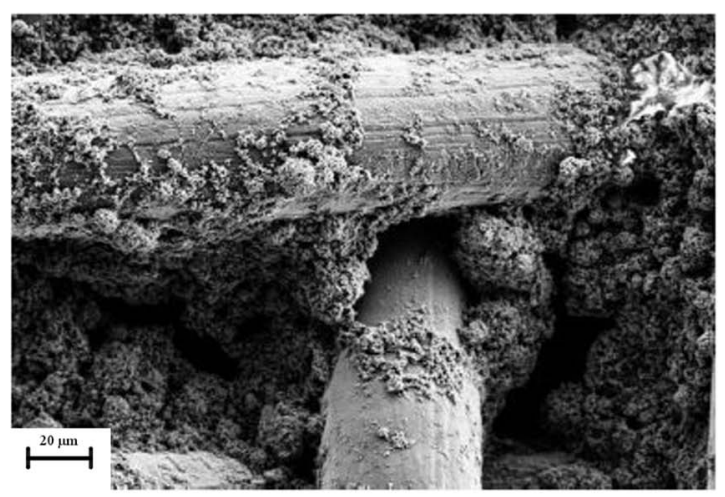

b)

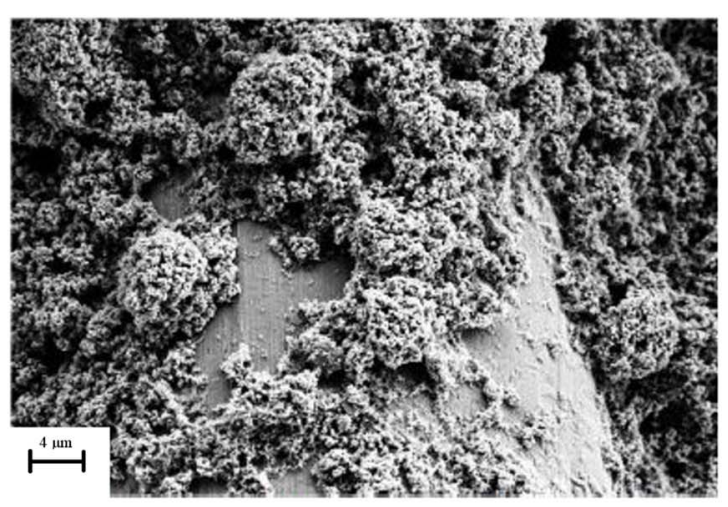

c)

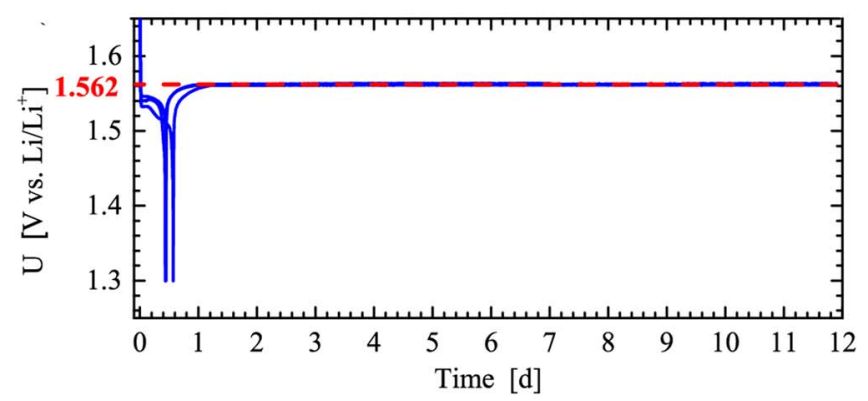

Figure 3. a),b) SEM pictures of LTO coating on Al-mesh. c) Potential of LTO coated Al-meshes during lithiation and relaxation in a coin cell against a lithium metal electrode. The cells are discharged (corresponds to lithiating the LTO electrodes) until 1.3 V. Afterwards no current is flowing and only the cell voltage is measured. 

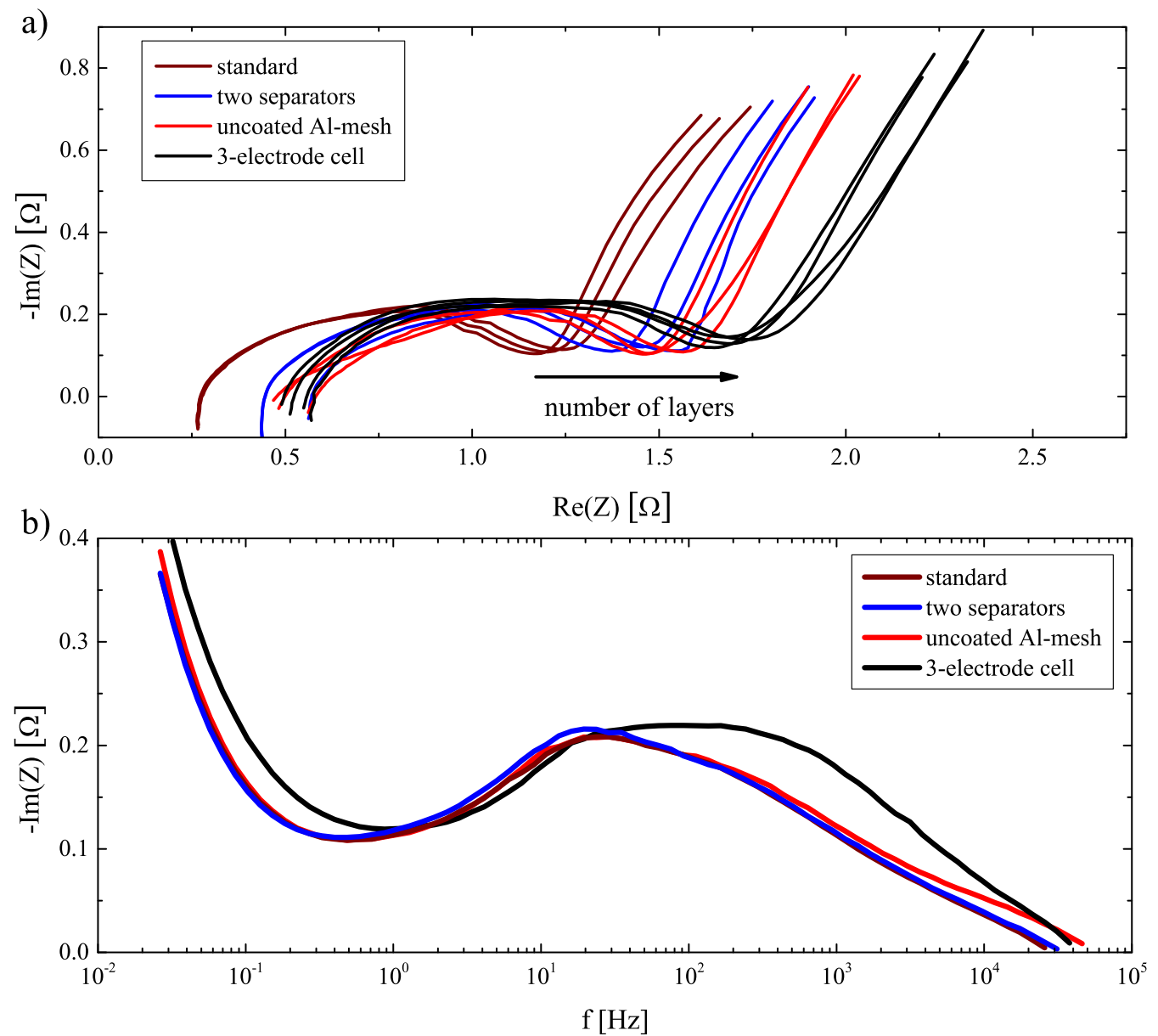

Figure 4. a) Impedance spectra of all tested cell designs in a Nyquist plot obtained at $3.6 \mathrm{~V}$ cell voltage (about $20 \%$ SoC). b) Plot of the imaginary part shown in a) vs. frequency to underline the deviation of the 3 -electrode cells at higher frequency.

by the manual fabrication. Still, the shape of the spectra in Figure 4a stays mostly the same for all cells indicating otherwise unchanged cell dynamics. This is additionally indicated by the very similar imaginary impedance curves in Figure 4b. Only the LTO 3-electrode cells show a small deviation from the rest in the frequency range from $100 \mathrm{~Hz}$ to $10 \mathrm{kHz}$. Since the 3 -electrode cells only differ in the LTO coating from the cells with uncoated aluminum meshes, this difference must be introduced by the LTO coating. In this frequency range capacitive effects dominate the impedance spectra so that additional electrochemical double layers might cause this deviation. ${ }^{27}$ Furthermore, Costard et al. have proposed a second lithium diffusion path between anode and cathode: A part of the lithium-ions do not travel through the pores of the reference electrode but are intercalated on the one side of the LTO coating and deintercalated on the other side. ${ }^{19}$ To limit this influence the use of a more open mesh with less LTO in the form of a very thin layer is planned. Nonetheless, with the present 3-electrode cells a detailed analysis of the cell materials and operating parameters is feasible.

Aging behavior of modified cells vs. standard cells.-The influence of the LTO reference electrode on the cell aging behavior was characterized by accelerated cyclic aging experiments. To this end, three standard cells and six 3-electrode cells were cycled at $45^{\circ} \mathrm{C}$ with $3 \mathrm{C}$ full cycles between $3 \mathrm{~V}$ and $4.15 \mathrm{~V}$. Before the cycling started and after 250 and 500 cycles the condition of each cell was characterized using a check-up test as described above.

Figure 5 shows the capacity retention of the cells normalized to the initial capacity (state of health, $\mathrm{SoH}$ ) as determined during the check-ups last $1 \mathrm{C}$ discharge. The absolute capacity of the 3 -electrode cells is about $1 \mathrm{mAh}$ smaller since part of the lithium is bound in the LTO to provide a stable reference potential. Additionally, the higher internal resistance of the LTO cells decreases the measured capacity as the voltage limits in charge and especially in discharge direction are reached earlier.

Already after 250 cycles the cell capacities start to scatter. It is known that the reproducibility of manually built lab cells is not perfect. Thus, a few outliers were expected. An analysis using box plots shows that one standard cell and two 3-electrode cells behave anomalously. These cells are not included in any further analyses.

Figure 5 demonstrates that the 3 -electrode cells lose more capacity over the course of the aging cycles. After 500 cycles the standard cells perform better and maintain about $85 \%$ of their initial capacity. The 3 -electrode cells retain about $75 \%$ of their initial capacity. This is likely caused by a higher diffusion resistance in the 3-electrode cells due to the longer distance between anode and cathode compared to the standard cells. A longer diffusion path in the electrolyte can lead to low lithium ion concentrations on the anodes surface during charging, which increases the charge transfer overpotential and thus the risk of lithium plating. Furthermore, an inhomogeneous compression of the cell due to the dip coated reference as well as interactions of the electrodes with the LTO reference material are possible causes. However, these influences are difficult to determine. Further decreasing the thickness of the reference mesh and making the LTO coating more homogenous will likely reduce the observed differences.

Stability of the LTO reference mesh.-A main goal of this study was to find a stable reference electrode which can remain inside the lithium ion cell while it undergoes aging tests. In order to verify that 

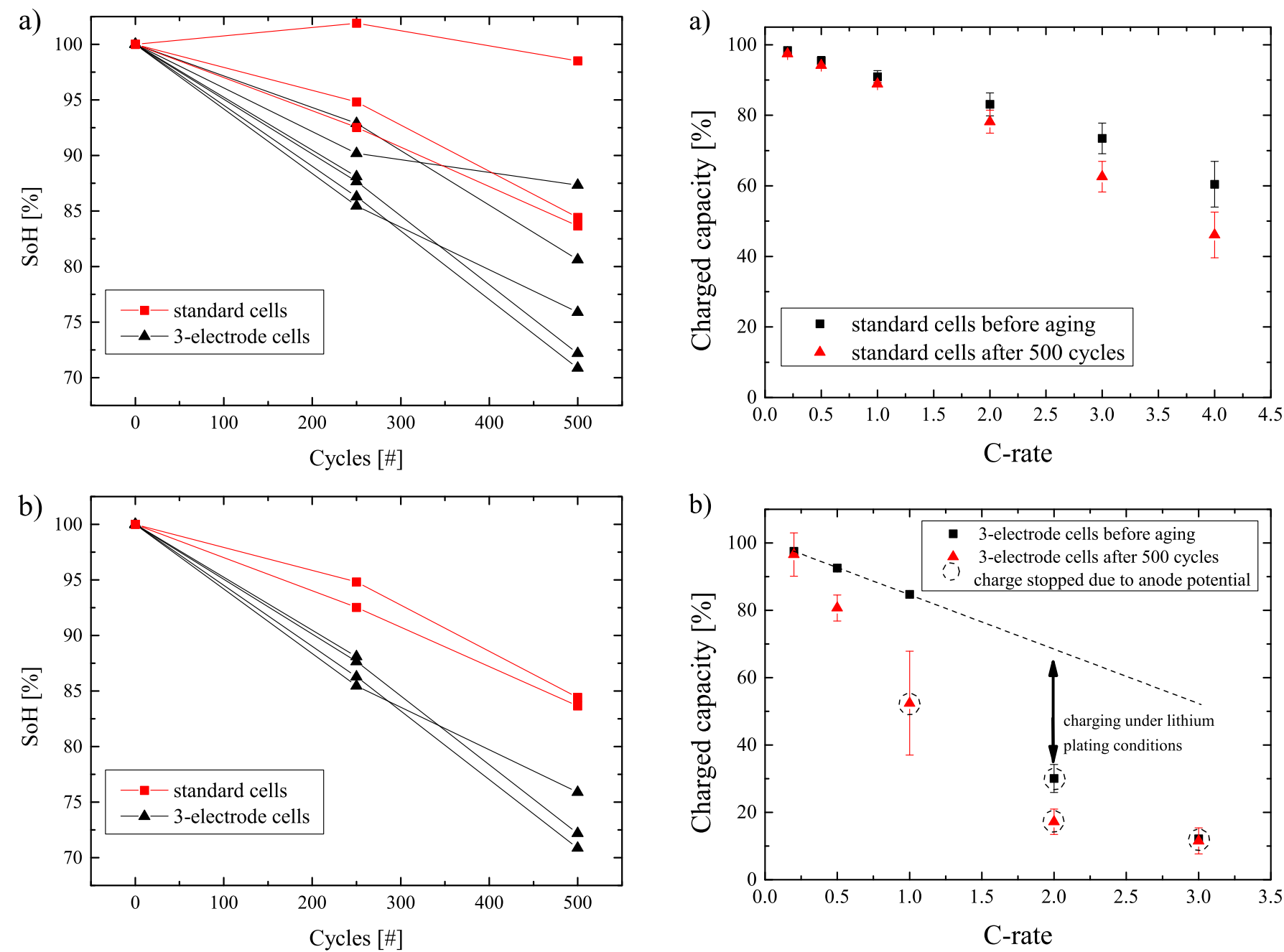

Figure 5. Development of the state of health of standard cells (red) and 3 -electrode cells (black) during continuous cycling at $45^{\circ} \mathrm{C}$ with $3 \mathrm{C}$ full cycles. In a) all tested cells are shown. In b) outliers as determined using box plots were removed. One 3-electrode cell was damaged during the check-up after 250 cycles.

this is indeed the case for the here described LTO coated aluminum mesh, several analyses were performed on fresh and aged reference meshes as obtained from opened cells. X-ray diffraction (XRD) as well as SEM imaging did not indicate any microscopic change of the utilized LTO. Furthermore, energy-dispersive X-ray spectroscopy (EDX) was used to look for titanium on the surface of the anode and cathode in aged cells but none was found. Finally, the reference electrodes did not show any loss of capacity and provided a stable reference potential after the aging cycling. However, for a stable reference potential, it was necessary to recharge the reference electrodes as the voltage measurement slowly drained the lithium from the LTO during aging cycling.

Charging capability: new cell vs. aged cell.-The capability to be charged quickly is tested using asymmetric C-rate tests where the cells were always fully discharged with the same current (1C) and then charged with varying currents. Due to the increasing overpotentials at higher currents, the charge throughput decreases since the cutoff voltage is reached earlier. Figure $6 \mathrm{a}$ shows the reachable $\mathrm{SoC}$ with varying constant currents when starting the charging process at $0 \%$ SoC for a standard cell. $100 \%$ SoC marks the $1 \mathrm{C}$ discharge capacity after a $1 \mathrm{C}$ charge step with $\mathrm{CV}$ phase to $\mathrm{C} / 20$ as determined in the preceding check-up. An almost linear decrease of the reachable SoC

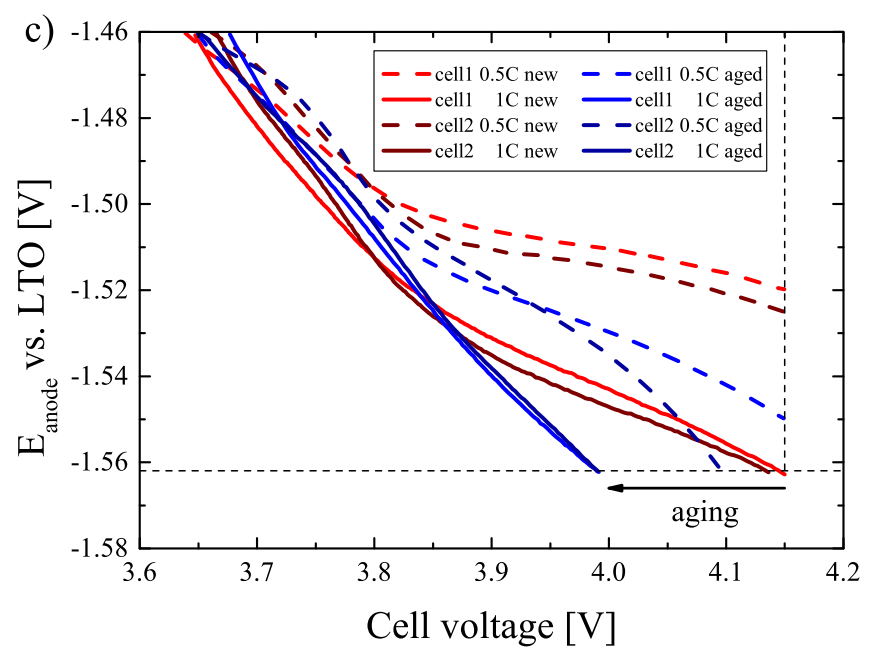

Figure 6. Achievable SoC during constant current charging until $4.15 \mathrm{~V}$ in standard cells a) and in 3-electrode cells b). For the 3-electrode cells the charging was additionally terminated if the anode potential fell below $-1.562 \mathrm{~V}$ vs. LTO. Charge steps which were terminated by the anode potential are marked with a circle. In c) the anode potential curves are plotted over the full cell potential of a 3-electrode cell before and after aging cycles. 
with rising C-rate before and after aging is observed. This is expected as the internal resistance of a cell can be described as a series of RC elements. At some point during continuous charging all capacities are fully charged and a series of ohmic resistances remains. ${ }^{27}$ After 500 aging cycles the achievable SoC for charging currents higher than $1 \mathrm{C}$ are slightly lower than for the fresh cell indicating an increased ohmic resistance.

For the 3-electrode cells another charge step termination criterion for the anode was introduced at $-1.562 \mathrm{~V}$ vs LTO $\left(0 \mathrm{~V}\right.$ vs. $\left.\mathrm{Li} / \mathrm{Li}^{+}\right)$in order to avoid lithium plating and thus additional damage. As shown in Figure 6b, this creates quite a different picture than for the standard cells. For a fresh 3-electrode cell the $\mathrm{CC}$ phase is terminated by the maximum cell voltage of $4.15 \mathrm{~V}$ up to a C-rate of $1 \mathrm{C}$. When charging with a current of $2 \mathrm{C}$ or higher, the charging is terminated due to the anode potential reaching the lithium plating criterion. Thus, only about $20 \%$ SoC is reached at $2 \mathrm{C}$. Linearly extrapolating the data up to $1 \mathrm{C}$ in Figure $6 \mathrm{~b}$ (dashed line) demonstrates that at $2 \mathrm{C}$ the cell could still be charged up to $70 \%$ SoC until the maximum cell voltage would be reached. However, from $30 \%$ SoC until $70 \%$ SoC at least a part of the charge would lead to lithium plating on the anode. After 500 aging cycles the critical charging rate, where the charge step is terminated by the full cell voltage and not by the anode potential, drops to $\mathrm{C} / 2$. In contrast to a fresh cell the aged cell cannot be charged with $1 \mathrm{C}$ to $4.15 \mathrm{~V}$ without causing lithium plating. This is demonstrated in Figure $6 \mathrm{c}$ where the anode potential is plotted against the full cell potential for two 3 -electrode cells in a fresh and aged state. Thus, the anode reaches the plating criterion while the cell voltage lags behind. An increasing susceptibility to lithium plating with aging has been theoretically predicted by Yang et al. who explained this with decreasing anode porosity due to SEI growth. ${ }^{22}$ Schuster et al. have speculated that aging under harsh conditions can lead to lithium plating even at moderate conditions and finally to selfenforcing capacity loss. ${ }^{28}$ The $\mathrm{C}$-rate tests shown here seem to confirm those theories. This leads to the conclusion that fast charging protocols which are developed for fresh lithium-ion cells explicitly to avoid lithium plating might suddenly produce lithium plating when the cell is aged.

Optimized charging procedure based on anode potential.-The aim of a fast charging protocol for any cell should be to minimize the charging time to a certain $\mathrm{SoC}$ while avoiding additional damage. Apart from temperature limits which must not be exceeded during cell operation, lithium plating is the main limitation for fast charging protocols for lithium-ion cells. ${ }^{9-11,29,30}$ The 3-electrode cells built here were used to investigate how much faster they can be charged under optimal current control conditions if compared to a standard CC-CV charging procedure. In order to achieve this, a step wise charging procedure was applied for the 3-electrode cells. According to this, the cells were charged at $23^{\circ} \mathrm{C}$ from $0 \%$ SoC with $4 \mathrm{C}$ until the anode potential reaches $0 \mathrm{~V}$ vs. $\mathrm{Li} / \mathrm{Li}^{+}$or if the maximum full cell voltage of $4.15 \mathrm{~V}$ is reached. After a 1 minute rest period to allow the cells to relax from higher overpotentials of the previous charge step with higher current, the charging continues with $3 \mathrm{C}$ and so on. The applied current steps were $4 \mathrm{C}, 3 \mathrm{C}, 2 \mathrm{C}, 1.5 \mathrm{C}, 1 \mathrm{C}$ and $0.5 \mathrm{C}$. Figure $7 \mathrm{a}$ shows which $\mathrm{SoC}$ is reached in a fresh cell with every $\mathrm{C}$-rate before the rest period starts. As the charging steps at high current are all terminated due to the anode potential reaching $0 \mathrm{~V}$ vs. $\mathrm{Li} / \mathrm{Li}^{+}$, the anode potential curve from a $\mathrm{C} / 20 \mathrm{CC}$ charge step is included, which was recorded before the step wise charging, on the right y-axis in Figure 7a. This seems appropriate since the overpotentials in a cell are approximately proportional to the internal cell resistance for continuous charging multiplied by the charge current according to Ohms law. A good correlation of the termination of the charging steps with the nearly OCV characteristic of the anode is observed. Thus, the step wise charging procedure appears to utilize the overpotential reserve (the difference between the anode open circuit voltage and $0 \mathrm{~V}$ vs. $\mathrm{Li} / \mathrm{Li}^{+}$) that the anode provides well. ${ }^{29}$

Figure $7 \mathrm{~b}$ shows the time it takes to reach a certain $\mathrm{SoC}$ with a $1 \mathrm{C} \mathrm{CC}$ charge (red dotted line) and with step wise charging. The step
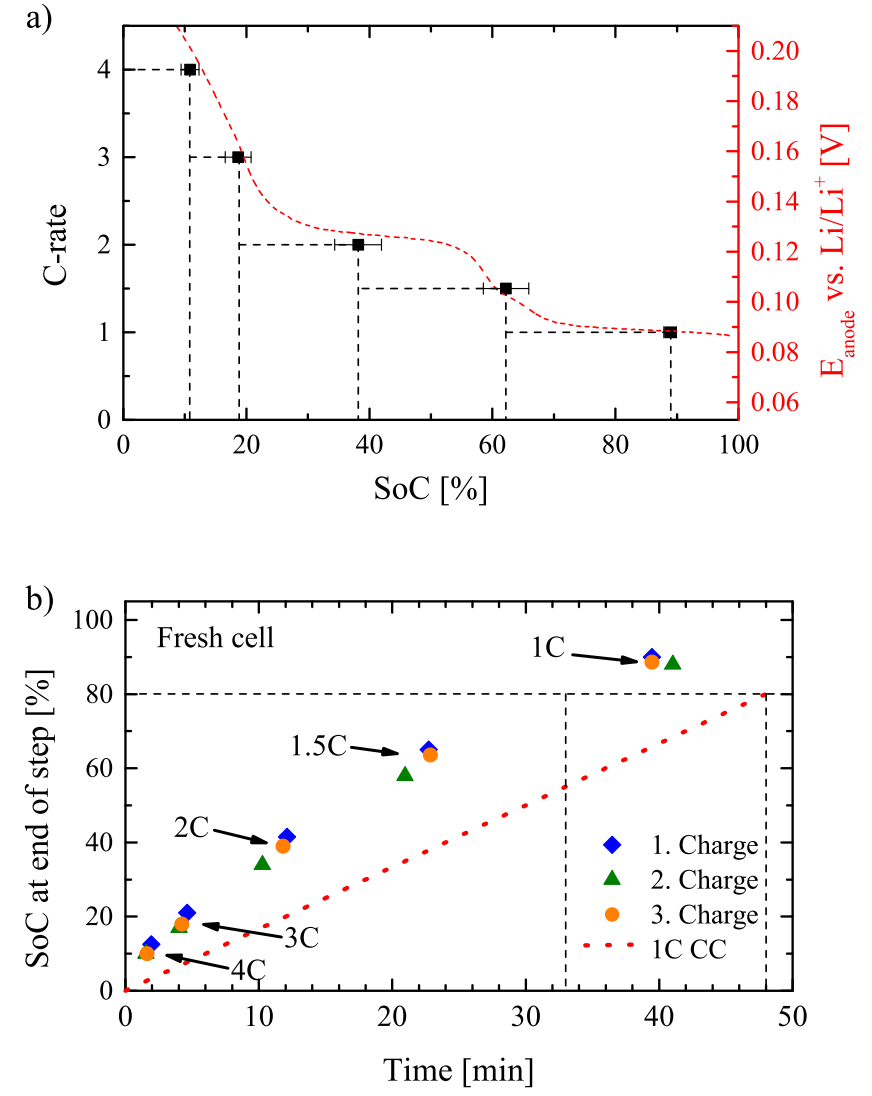

c)

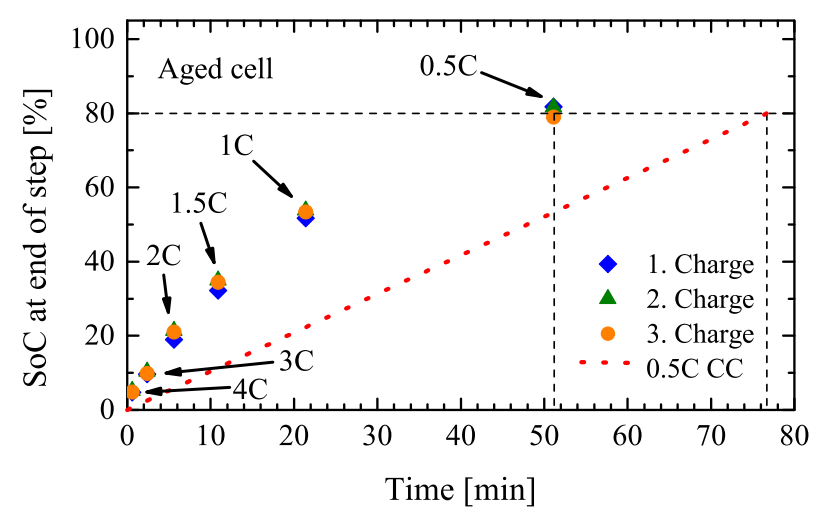

Figure 7. Step wise charging of 3-electrode cells. Current steps of a fresh cell are shown in a) as well as the anode potential from a C/20 CC charge. In b) the time it takes to charge to a certain SoC is shown for all charging steps for a fresh cell. In c) the same graph is shown for an aged cell. The red dotted lines show the charging curves of a $1 \mathrm{C} \mathrm{CC}$ charge in b) respectively a $0.5 \mathrm{C}$ $\mathrm{CC}$ charge in c) as a comparison. Dashed lines are included to guide the eye. Results are shown for three consecutive charging steps. Also note the different $\mathrm{x}$-axis in $\mathrm{b}$ ) and c). An overview over the achieved charging times is given in Table II.

wise charging is compared to a $1 \mathrm{C} \mathrm{CC}$ charge as $1 \mathrm{C}$ is the maximum constant current which allows the cell to be charged from $0 \% \mathrm{SoC}$ to $80 \%$ SoC without switching to constant voltage phase. An SoC of $80 \%$ is chosen as the target SoC for the fast charging protocol as it is often communicated by electric car manufacturers. In the case of the 3 -electrode cells, a reduction in charging time to $80 \%$ SoC from $48 \mathrm{~min}$ to approximately $33 \mathrm{~min}$ was achieved when charging with the step wise charging protocol compared to $1 \mathrm{C} \mathrm{CC}$ charging protocol. This 


\begin{abstract}
Table II. Overview of achievable charging times to $80 \%$ SoC in fresh and aged 3-electrode cells during step wise charging or constant current charging with maximum current without lithium plating.
\end{abstract}

\begin{tabular}{ccc} 
Charging time to $80 \%$ SOC & fresh cell [min] & aged cell [min] \\
\hline Step charging & 33 & 51 \\
Constant current & 48 & 77
\end{tabular}

corresponds to a reduction of about $30 \%$. A summary of the achieved charging times is shown in Table II.

Further improvements in charging time without additional damage are only achievable by increasing the temperature during charging to speed up cell kinetics or by improving the cell chemistry. In terms of current control strategy, we believe a step wise charging which maximizes the utilization of the anode potential curve at every given temperature, is the best possible way. Moreover, if a shorter charging time or lower SoC is desired, the step wise charging protocol is even more advantageous, e.g. after a charging time of 20 min the SoC can approximately be doubled.

In order to demonstrate the influence of increasing overpotentials on the anode side due to aging, Figure $7 \mathrm{c}$ shows the results of the step wise charging on an aged cell. The charging to $80 \%$ SoC (relative to remaining capacity) with the step wise charging protocol now takes about 51 minutes. Another charging step with $0.5 \mathrm{C}$ is needed to reach the desired $\mathrm{SoC}$ as the foregoing $1 \mathrm{C}$ is terminated early at only $55 \%$ SoC as the anode potential reaches $0 \mathrm{~V}$ vs. $\mathrm{Li} / \mathrm{Li}^{+}$. Still, the step wise charging protocol is much faster than a $0.5 \mathrm{C}$ constant current charge which takes about 77 minutes to reach $80 \%$ SoC (see Table II). Also after a charging time of $20 \mathrm{~min}$ the difference is more pronounced as for the fresh cell: With the step wise charging protocol a SoC of more than $50 \%$ can be achieved compared to about $20 \%$ of $1 \mathrm{C} \mathrm{CC}$ charging.

This work shows that special care has to be taken when defining such fast charging procedures. Safety margins which take the aging of the cell into account should be included as this would increase cell lifetime. Alternatively, the charging protocol has to be dynamically adapted to the state of health.

\section{Conclusions}

In this study 3 -electrode cells are proving to be a valuable tool to investigate and understand state of the art lithium-ion cells. Aluminum meshes coated with LTO allow the production of durable pouch cells which can undergo performance and aging tests. However, EIS and aging investigations show that a direct comparison of these 3-electrode cells with standard cells is challenging. The longer distance between anode and cathode as well as the porous LTO coating increase the internal resistance of the cells. This leads to accelerated aging of the 3 -electrode cells. In order to improve the transferability of results gathered from 3-electrode cells, the thickness of the additional layers should be reduced as well as the coating of the aluminum mesh. A sparse expanded aluminum mesh coated with LTO nanoparticles and very thin separators should allow a better transfer of results from the 3-electrode to the standard cells.

Here, the focus were fundamental performance studies. C-rate tests were used to deduce a step wise charging protocol which optimizes the use of the anode overpotential reserve while avoiding additional damage due to lithium plating. A reduction of the charging time of about $30 \%$ could be achieved to reach $80 \%$ SoC for fresh cells compared to a standard constant current charge. However, aged 3-electrode cells show an increased susceptibility to lithium plating as the anode overpotentials increase during aging. Using the protocol which was optimized for the fresh cells would lead to lithium plating in aged cells. Thus, the step wise charging protocol had to be adapted for the aged cells. With the adapted step wise charging protocol the achievable SoC can be more than doubled after a charging time of $20 \mathrm{~min}$.

\section{Acknowledgments}

The results, opinions and conclusions expressed in this thesis are not necessarily those of Volkswagen Aktiengesellschaft.

\section{ORCID}

Bernd Epding (D) https://orcid.org/0000-0002-2330-3042

\section{References}

1. H. Zheng, J. Li, X. Song, G. Liu, and V. S. Battaglia, "A comprehensive understanding of electrode thickness effects on the electrochemical performances of Li-ion battery cathodes," Electrochimica Acta, 71, 258 (2012).

2. P. Arora, "Mathematical Modeling of the Lithium Deposition Overcharge Reaction in Lithium-Ion Batteries Using Carbon-Based Negative Electrodes," Journal of The Electrochemical Society, 146(10), 3543 (1999).

3. T. Waldmann, B.-I. Hogg, and M. Wohlfahrt-Mehrens, "Li plating as unwanted side reaction in commercial Li-ion cells - A review," Journal of Power Sources, 384, 107 (2018).

4. T. Waldmann, B.-I. Hogg, M. Kasper, S. Grolleau, C. G. Couceiro, K. Trad, B. P. Matadi, and M. Wohlfahrt-Mehrens, "Interplay of Operational Parameters on Lithium Deposition in Lithium-Ion Cells," Journal of The Electrochemical Society, 163(7), A1232 (2016).

5. N. Legrand, B. Knosp, P. Desprez, F. Lapicque, and S. Raël, "Physical characterization of the charging process of a Li-ion battery and prediction of $\mathrm{Li}$ plating by electrochemical modelling," Journal of Power Sources, 245, 208 (2014).

6. M. Petzl and M. A. Danzer, "Nondestructive detection, characterization, and quantification of lithium plating in commercial lithium-ion batteries," Journal of Power Sources, 254, 80 (2014).

7. S. Schindler, M. Bauer, M. Petzl, and M. A. Danzer, "Voltage relaxation and impedance spectroscopy as in-operando methods for the detection of lithium plating on graphitic anodes in commercial lithium-ion cells," Journal of Power Sources, 304, 170 (2016).

8. J. C. Burns, D. A. Stevens, and J. R. Dahn, "In-Situ Detection of Lithium Plating Using High Precision Coulometry," Journal of the Electrochemical Society, 162(6), A959 (2015).

9. T. Waldmann, M. Kasper, and M. Wohlfahrt-Mehrens, "Optimization of Charging Strategy by Prevention of Lithium Deposition on Anodes in high-energy Lithiumion Batteries - Electrochemical Experiments," Electrochimica Acta, 178, 525 (2015).

10. T. Amietszajew, E. McTurk, J. Fleming, and R. Bhagat, "Understanding the limits of rapid charging using instrumented commercial 18650 high-energy Li-ion cells," Electrochimica Acta, 263, 346 (2018).

11. S. S. Zhang, K. Xu, and T. R. Jow, "Study of the charging process of a $\mathrm{LiCoO}_{2}$-based Li-ion battery," Journal of Power Sources, 160(2), 1349 (2006).

12. V. Zinth, C. Lüders, M. Hofmann, J. Hattendorff, I. Buchberger, S. Erhard, J. Rebelo-Kornmeier, A. Jossen, and R. Gilles, "Lithium plating in lithium-ion batteries at sub-ambient temperatures investigated by in situ neutron diffraction," Journal of Power Sources, 271, 152 (2014).

13. B. Bitzer and A. Gruhle, "A new method for detecting lithium plating by measuring the cell thickness," Journal of Power Sources, 262, 297 (2014).

14. J. Wandt, P. Jakes, J. Granwehr, R.-A. Eichel, and H. A. Gasteiger, "Quantitative and time-resolved detection of lithium plating on graphite anodes in lithium ion batteries,' Materials Today, 21(3), 231 (2018).

15. S. A. Kayser, A. Mester, A. Mertens, P. Jakes, R.-A. Eichel, and J. Granwehr, "Longrun in operando NMR to investigate the evolution and degradation of battery cells," Physical chemistry chemical physics: PCCP, (2018).

16. M. Ender, J. Illig, and E. Ivers-Tiffée, "Three-Electrode Setups for Lithium-Ion Batteries," Journal of The Electrochemical Society, 164(2), A71 (2016).

17. G. Nagasubramanian and D. H. Doughty, "18650 Li-ion cells with reference electrode and in situ characterization of electrodes," Journal of Power Sources, 150, 182 (2005).

18. J. R. Belt, D. M. Bernardi, and V. Utgikar, "Development and Use of a LithiumMetal Reference Electrode in Aging Studies of Lithium-Ion Batteries," Journal of the Electrochemical Society, 161(6), A1116 (2014).

19. J. Costard, M. Ender, M. Weiss, and E. Ivers-Tiffée, "Three-Electrode Setups for Lithium-Ion Batteries," Journal of The Electrochemical Society, 164(2), A80 (2016).

20. M. Dollé, F. Orsini, A. S. Gozdz, and J.-M. Tarascon, "Development of Reliable Three-Electrode Impedance Measurements in Plastic Li-Ion Batteries," Journal of The Electrochemical Society, 148(8), A851 (2001).

21. F. La Mantia, C. D. Wessells, H. D. Deshazer, and Y. Cui, "Reliable reference electrodes for lithium-ion batteries," Electrochemistry Communications, 31, 141 (2013).

22. X.-G. Yang, Y. Leng, G. Zhang, S. Ge, and C.-Y. Wang, "Modeling of lithium plating induced aging of lithium-ion batteries," Journal of Power Sources, 360, 28 (2017). 
23. K. Jalkanen, J. Karppinen, L. Skogström, T. Laurila, M. Nisula, and K. Vuorilehto, "Cycle aging of commercial NMC/graphite pouch cells at different temperatures," Applied Energy, 154, 160 (2015).

24. M. Ecker, P. Shafiei Sabet, and D. U. Sauer, "Influence of operational condition on lithium plating for commercial lithium-ion batteries - Electrochemical experiments and post-mortem-analysis," Applied Energy, 206, 934 (2017).

25. S. J. An, J. Li, Z. Du, C. Daniel, and D. L. Wood, "Fast formation cycling for lithium ion batteries," Journal of Power Sources, 342, 846 (2017).

26. M. Reichert, D. Andre, A. Rösmann, P. Janssen, H.-G. Bremes, D. U. Sauer, S. Passerini, and M. Winter, "Influence of relaxation time on the lifetime of commercial lithium-ion cells,” Journal of Power Sources, 239, 45 (2013).
27. U. Tröltzsch, O. Kanoun, and H.-R. Tränkler, "Characterizing aging effects of lithium ion batteries by impedance spectroscopy," Electrochimica Acta, 51(8-9), 1664 (2006).

28. S. F. Schuster, T. Bach, E. Fleder, J. Müller, M. Brand, G. Sextl, and A. Jossen, "Nonlinear aging characteristics of lithium-ion cells under different operational conditions," Journal of Energy Storage, 1, 44 (2015).

29. S. Schindler and M. A. Danzer, "A novel mechanistic modeling framework for analysis of electrode balancing and degradation modes in commercial lithium-ion cells," Journal of Power Sources, 343, 226 (2017).

30. P. Keil and A. Jossen, "Charging protocols for lithium-ion batteries and their impact on cycle life-An experimental study with different 18650 high-power cells," Journal of Energy Storage, 6, 125 (2016). 